\title{
Special issue in flow chemistry
}

\author{
György M. Keserú · György Dormán
}

Received: 28 June 2011 / Accepted: 29 June 2011 / Published online: 13 July 2011

(C) Springer Science+Business Media B.V. 2011

In the last decade, we have witnessed a steady transformation of organic chemistry. The number of launched new chemical entities decreased in spite of the number of synthetically accessible small molecules that exponentially increased. On the other hand, the drug like chemical space was only poorly sampled and the huge compound decks at Big Pharma companies were saturated with redundant structures. As a consequence, new synthetic techniques were required and emerged which were collectively called "enabling chemistry" [1]. Enabling chemistry methods contribute to accelerate chemical processes, explore new chemistries, increase the throughput and facilitate the workup and isolation. Such processes also meet several criteria of Green chemistry (i.e., increased reaction rate, reducing waste, and increased atom economy) and contribute to the development of sustainable manufacturing (i.e., recycling, biomass transformation, etc.). Enabling chemistry led the way to continuous flow chemistry and microreactors [2]. While the significant advancement in immobilized catalysts and supported reagents naturally found application in continuous flow systems (i.e., fixedbed microreactors) the wide-spread application of microwave-assisted organic synthesis (MAOS) promoted the further expansion of the boundaries of the chemical parameter space [3]. However, the batch nature of synthetic organic chemistry and the chemical parameter space it covers has not changed during the ca. 200 years' history of organic synthesis and only a small fraction of the parameter space is covered

G. M. Keserû $(\varangle)$

Discovery Chemistry, Gedeon Richter plc., 19-21 Gyömrői út, Budapest 1103, Hungary

e-mail: gy.keseru@ richter.hu

\section{G. Dormán}

ThalesNano Nanotechnology Inc., Záhony u. 7., Budapest 1031, Hungary

e-mail: gyorgy.dorman@thalesnano.com
(0-10 bar, -78 to $250^{\circ} \mathrm{C}$ ) and reactions are still difficult to fully optimize under the usual time and resource constraints.

Flow (micro) reactors are now possible to access significantly higher temperature and pressure parameters (presently up to $200 \mathrm{bar}, 350^{\circ} \mathrm{C}$ ); allows rapid optimization and scaleup; enables increased heat and mass transfer. Using fixed-bed reactors the products leave the reaction zone to avoid side reactions. High selectivity can be achieved with residence time control and since the reaction zone is relatively small highly exothermic and hazardous reactions can be performed under normal laboratory environment. All these advantages contribute to the fast and significant increase of novel flow processes and the number of publications in the recent years. This rapid penetration into the synthetic laboratory practice justified the present Special Issue in Molecular Diversity which includes four publications covering various areas and application of this emerging discipline.

Baumann et al. provides a comprehensive account on the continuous flow synthesis of heterocycles for natural product and medicinal chemistry applications. This overview is based on his earlier presentation hold at the FROST2 meeting on "Recent Advances on Flow Chemistry" in Budapest in October 2009. The article describes the synthesis of various heteroaromatic small molecule libraries synthesized by flow techniques through the combination of various instruments, configurations and setups with a broad literature support.

Mandity et al. describes an elegant application of a fixedbed continuous flow hydrogenator in catalytic deuteration. Deuterated compounds gained much interest in NMR and MS studies, where high isotopic purity is required. The article demonstrates through the deuteration of several nitrogen containing heterocyclic model compounds that flow techniques can be the method of choice lacking most of the drawbacks of the conventional batch deuteration techniques. 
Mihovilovic et al. presents here an excellent example that flow systems can successfully be exploited in sustainable chemistry. One of the challenges in this area is how to produce important fine chemicals from renewable natural sources. In order to find the appropriate parameters and optimize such transformations rapidly flow devices could be the beneficial. This article describe the optimization of dehydration of fructose and glucose in dipolar, aprotic solvents to 5-hydroxymethylfurfural. Various heating methods were compared including conventional, thermal heating, and stopflow microwave heating conditions and residence time/ reaction time parameters were optimized.

Lengyel et al. describes a high temperature/pressure method for a stepwise aromatic nucleophilic substitution of difluoro-benzenes having an activating group in meta position to the fluorines. Using a resistance-heated homogeneous coil flow reactor they prepared an unsymmetrically substituted 3,5-diamino-benzonitrile library in high yield and selectivity in an extremely short reaction time, compared with the conventional batch or microwave technique.

\section{References}

1. Kirschning A, Solodenko W, Mennecke K (2006) Comining enabling techniques in organic synthesis-continuous flow processes with heterogenized catalysts. Chem Eur J 12:5972-5990. doi:10.1002/chem.200600236

2. Wirth T (2008) Microreactors in organic synthesis and catalysis. Wiley-VCH, Weinheim

3. Darvas F, Dormán G, Lengyel L, Kovács I, Jones R, Ürge L (2009) High pressure, high temperature reactions in continuous flow.Merging discovery and process chemistry. Chim Oggi 27:36140-36143 